STEREOTYPE THREAT \& INHIBITORY CONTROL

The Effects of Stereotype Threat and Contextual Cues on Alcohol Users' Inhibitory Control

Word Count: 3533

Charlotte R. Pennington ${ }^{1 *}$, Adam Qureshi ${ }^{1}$, Rebecca L. Monk ${ }^{1}$ \& Derek Heim ${ }^{1}$ ${ }^{1}$ Department of Psychology, Edge Hill University, Ormskirk, Lancashire.

*Corresponding Author: Charlotte Pennington, Department of Psychology, Edge Hill University, St Helens Road, Ormskirk, Lancashire, United Kingdom, L39 4QP. E-mail: penninc@edgehill.ac.uk

Key words: STEREOTYPE THREAT; ALCOHOL; EXPECTANCIES;

INHIBITORY CONTROL; EXECUTIVE FUNCTION 


\begin{abstract}
Aim: Previous research indicates that users of illicit substances exhibit diminished cognitive function under stereotype threat. Advancing this research, the current study aimed to examine the effects of stereotype threat on alcohol users' inhibitory control. It also examined whether drinkers' demonstrate a greater approach bias towards alcohol-related relative to neutral stimuli. Method: Fifty-five participants were assigned randomly to a stereotype threat condition, in which they were primed with a negative stereotype linking drinking behavior to cognitive decline, or a control condition. All participants then completed a modified version of the Cued Go/No-Go Association Test that exposed participants to alcohol-related and neutral pictorial stimuli and sound cues. Results: Stereotype threatened participants demonstrated a speed-accuracy trade off, taking significantly longer to respond to go-trials with equivalent accuracy to the control condition. They also showed reduced response accuracy to both alcohol-related and neutral stimuli in reversed instruction trials. Participants in the control condition were both more accurate and quicker to respond to alcohol-related stimuli compared to neutral stimuli. Conclusion: These results suggest that awareness of negative stereotypes pertaining to alcohol-related impulsivity may have a harmful effect on inhibitive cognitive performance. This may have implications for public health campaigns and for methodological designs with high levels of procedural signaling with respect to not inadvertently inducing stereotype threat and impacting impulsivity.
\end{abstract}


STEREOTYPE THREAT \& INHIBITORY CONTROL

\section{The Effects of Stereotype Threat and Contextual Cues on Alcohol Users' Inhibitory Control}

\section{Introduction}

Alcohol-dependent and heavy social drinkers often report an increase in subjective craving and demonstrate significant physiological arousal in response to alcohol-related cues (Field \& Cox, 2008; Field \& Duka, 2002). The link between alcohol consumption and cue reactivity has been attributed to the effects of alcohol on executive functioning, specifically diminished inhibitory control (Adams, Ataya, Attwood, \& Munafò, 2013). This body of work suggests that alcohol-related cues can function as a prime that decreases individuals' ability to inhibit a dominant response by suppressing behavioral impulses (Hogarth et al., 2012). Consistent with this prediction, Kreusch et al. (2013) found that both problem and non-problem drinkers made significantly more commission errors (i.e., false alarms) and were quicker to respond to alcohol-related stimuli compared to neutral stimuli in a Cued Go/No-Go Association Task. Other research has examined this notion in the more naturalistic setting of a simulated bar lab, indicating that alcohol-related cues may also increase subjective craving for alcohol and consumption (Jones et al., 2013). Indeed, the cognitions that are associated with increases in alcohol consumption may be contextually dependent (Monk \& Heim, 2013; Wall et al., 2000). As such, contextually salient alcohol-related cues may elicit a greater approach bias among drinkers, which may be associated with difficulty inhibiting behavioral responses (Fleming \& Bartholow, 2014; Rose \& Duka, 2008).

From an alternative point of view, it could be suggested that the mere salience of alcohol-related cues may create a situational burden in which individuals perceive that they will conform to a negative stereotype regarding their drinking behavior. 
Alcohol-related stereotypes may be particularly pertinent in the minds of alcohol drinkers, given that public health campaigns frequently disseminate information regarding the negative outcomes of alcohol consumption and its link to cognitive decline (Drink Aware, 2014; Feldstein-Ewing et al., 2014). However, it is not clear whether such knowledge manifests in behavioural changes for those who identify with this targeted group of individuals. From a theoretical perspective, stereotype threat suggests that this may be possible (Steele \& Aronson, 1995). Here, research shows that the situational salience of a negative stereotype may deplete working memory efficiency to bring about performance decrements on a given task (Schmader \& Johns, 2003). Conversely, other researchers have argue that the experience of stereotype threat may motivate individuals to disprove the negative stereotype, thus influencing goal engagement and enhanced control (Jamieson \& Harkins, 2007). Whilst not hitherto applied to alcohol consumption, research has examined the effects of stereotype threat on ecstasy and cannabis users' executive function performance (Cole et al., 2005; Looby \& Earleywine, 2010). It is therefore conceivable that the social stigma ascribed to alcohol-related cognitive inferiority may be a significant predictor of poor performance on tests of impulsivity.

In the first of its kind, the current study aims to investigate the influence of stereotype threat on alcohol users' inhibitory control. It is predicted that individuals who are primed with a negative stereotype regarding the perceived association between alcohol consumption and impulsivity will show heightened dis-inhibitory control relative to a control condition. Furthering this, the current study also examines the contention that inhibitory mechanisms of selective attention, implemented in drinking behaviors, are particularly susceptible to contextual influences. In line with 
STEREOTYPE THREAT \& INHIBITORY CONTROL

Kreusch et al. (2013) it is predicted that exposure to alcohol-related pictorial stimuli may reduce inhibitory control compared to neutral stimuli.

\section{Method}

\subsection{Participants}

Ethical approval was obtained at the institution where this study was carried out. Fifty-five participants (30 male; Mage $=24.20, S D=5.28 ; 92.9 \%$ White British) were recruited via an online participation website and received $£ 5(\$ 7.85)$ remuneration. Participants were randomly assigned to either the stereotype threat condition or the control condition and we not under the influence of alcohol at the time of testing. Average AUDIT scores were $11.07(\mathrm{SD}=6.99)$, which is comparable to recent research using UK student samples (Clarke, Field, \& Rose, 2015).

\subsection{Measures}

2.2.1. Cued Go-No/Go Association Task (GNAT). The strength of behavioral inhibition was assessed using a modified version of the Cued Go/No-Go task (Fillmore, 2003; Nosek \& Banaji, 2001). The current study utilized both alcoholrelated and neutral pictorial stimuli to examine the effects of contextual factors on response inhibition. In the experimental target condition, a picture of a beer bottle was used as alcohol-related stimuli and was contrasted with a water bottle. In the control target condition, alphabetical letters were employed as neutral stimuli and were contrasted with the letter ' $\mathrm{K}$ '. Before the start of each block, an instruction screen appeared on the computer, indicating which stimuli would be the go-target and which stimuli would be the no-go target. Standard instructions required participants to facilitate a response to go targets (water bottle; alphabetical letters) and to inhibit a response to no-go targets (beer bottle; letter ' $\mathrm{K}$ '). Reverse instructions required 


\section{STEREOTYPE THREAT \& INHIBITORY CONTROL}

participants to facilitate a response to no-go targets and inhibit a response to gotargets. In addition, participants also heard either a bar-related sound (i.e., the 'clinking/clashing' of bottles), which cued their responses to the pictorial stimuli, or no sound ${ }^{1}$.

Participants completed two short practice blocks of the GNAT followed by 16 critical blocks. Each block contained 70 trials, of which the initial five were discarded from analyses as practice trials. Each target was presented individually and alcoholrelated and neutral targets were presented in separate blocks. Pictorial targets were presented following an onset asynchrony of $500 \mathrm{~ms}$, remaining on the computer screen until a correct response was made (or up to a maximum 500ms). A central fixation cross was displayed for $500 \mathrm{~ms}$ and preceded the target on each trial. Auditory cues occurred randomly for a short interval on $50 \%$ of trials $(48 \mathrm{kHz})$. Table 1 shows the prevalence of stimuli type within each block. Figure 1 presents a schematic exemplar of the trial procedure.

Table 1. Target probabilities as a function of standard and reverse instruction blocks.

\begin{tabular}{|c|c|c|c|c|c|c|c|c|}
\hline \multirow{4}{*}{ Cue Type } & \multicolumn{4}{|c|}{ Standard instructions } & \multicolumn{4}{|c|}{ Reversed instructions } \\
\hline & \multicolumn{2}{|c|}{ Go Targets } & \multicolumn{2}{|c|}{ No-Go Targets } & \multicolumn{2}{|c|}{ Go Targets } & \multicolumn{2}{|c|}{ No-Go Targets } \\
\hline & Bar & No & Bar & None & Bar & No & Bar & No \\
\hline & Sound & Sound & Sound & Sound & Sound & Sound & Sound & Sound \\
\hline Alcohol & \multicolumn{2}{|c|}{.80} & \multicolumn{2}{|c|}{.20} & \multicolumn{2}{|c|}{.20} & \multicolumn{2}{|c|}{.80} \\
\hline Neutral & \multicolumn{2}{|c|}{.20} & \multicolumn{2}{|c|}{.80} & \multicolumn{2}{|c|}{.80} & \multicolumn{2}{|c|}{.20} \\
\hline
\end{tabular}

\footnotetext{
${ }^{1}$ A pilot study $(n=66)$ was conducted which also included a neutral sound cue (a supermarket background sound). Findings indicated that the neutral sound affected responding differentially to both alcohol-related and no sound, suggesting that different cues have different effects on inhibitory control. In the current study, we removed the supermarket sound to maximize any potential differences between alcohol sounds and no sound whilst also limiting the testing time.
} 


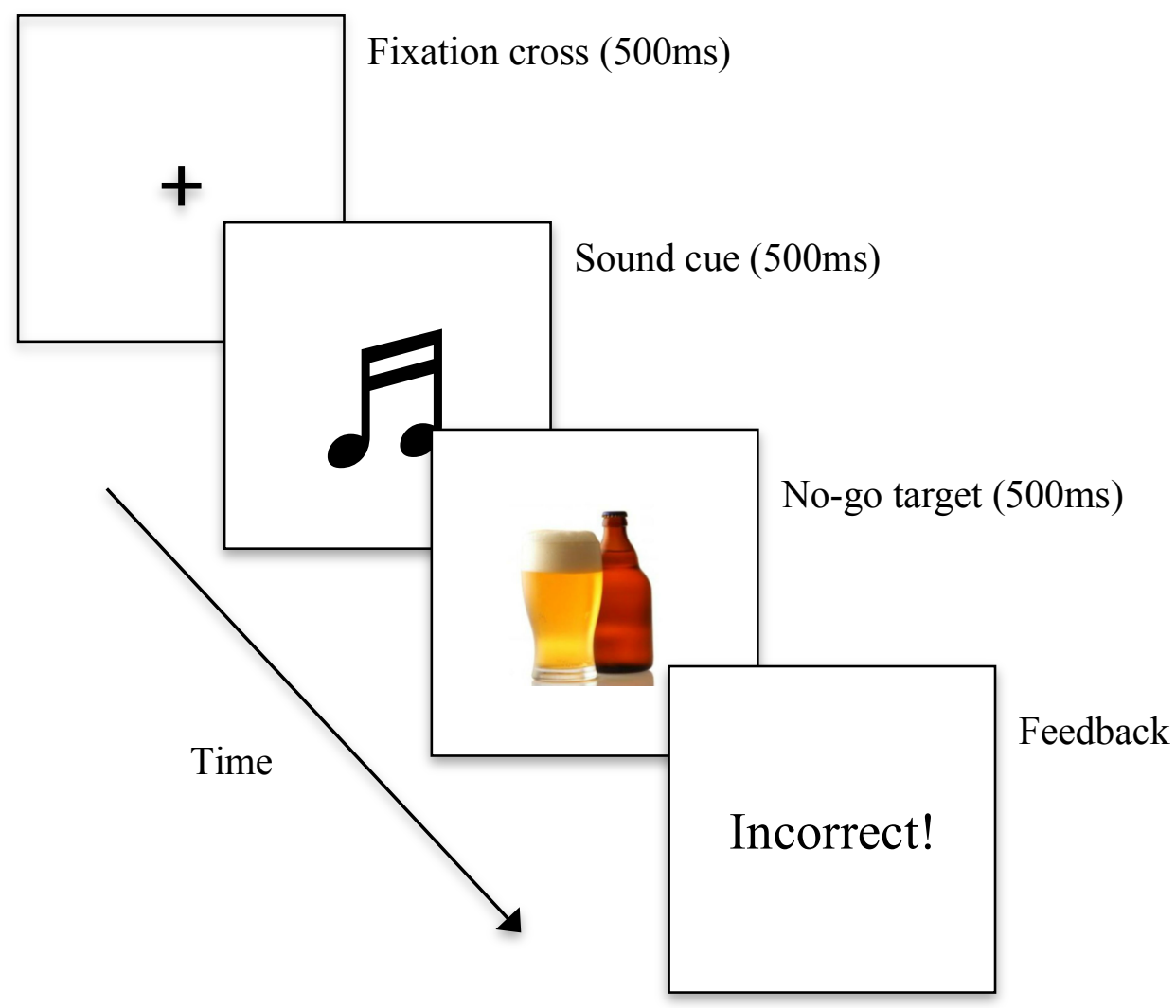

Figure 1. Example of an initiated incorrect response during a no-go trial.

2.2.2. Alcohol use Disorders Identification Test (AUDIT). The AUDIT

(Saunders et al., 1993) was utilized to ensure that there were no differences in typical alcohol consumption between the stereotype threat and control condition. This 10item screening questionnaire includes 4 sub-scales that assess patterns of alcohol consumption, drinking behavior, adverse reactions and problem drinking. Responses are recorded on a 5 -point Likert scale $(0=$ Never, $4=4$ or more times $)$. The AUDIT resulted in high internal consistency in the present sample, Cronbach's $a=.85$.

2.2.3. Adult Temperament Questionnaire (ATQ). The effortful control subscale of the ATQ (Rothbart et al., 2000) was utilized to ensure that there were no differences in self-reported executive control between experimental conditions. This sub-scale includes 35-items which measure sub-components of attentional control, 
STEREOTYPE THREAT \& INHIBITORY CONTROL

inhibitory control, and activation control. Responses are recorded on a 7-point Likert scale $(1=$ Extremely untrue of you, $7=$ Extremely true of you $)$. Each sub-scale resulted in high internal consistency, Cronbach's $a>.70$. See Table 2 for participant demographics on these measures.

Table 2. Measures of alcohol consumption and temperament as a function of experimental condition.

Stereotype Threat Control

\begin{tabular}{lcc}
\hline & \multicolumn{2}{c}{ Mean (SD) } \\
\hline Age & $23.71(6.08)$ & $24.50(4.79)$ \\
\hline AUDIT & $10.14(7.64)$ & $11.65(6.61)$ \\
\hline Inhibitory Control & $4.42(.85)$ & $4.12(.77)$ \\
\hline Activation Control & $4.52(1.16)$ & $4.56(.89)$ \\
\hline Attentional Control & $4.01(1.20)$ & $4.00(.84)$ \\
\hline Total Effortful Control & $4.32(.84)$ & $4.23(.60)$ \\
\hline
\end{tabular}

\subsection{Procedure}

Participants were assigned randomly to either the stereotype threat or the control condition. They were seated individually at a computer and were instructed to complete two practice blocks of the Go/No-Go Association Task (GNAT). Before progressing on to critical trials, the experimenter then implemented the stereotype threat manipulation. Participants in the stereotype threat condition were given additional information that linked drinking behavior with diminished inhibitory control. Here they read the following information:

Research suggests that alcohol consumption is linked to cognitive deficits. Specifically, people who use alcohol are more impulsive, and therefore are 
STEREOTYPE THREAT \& INHIBITORY CONTROL

less likely to inhibit behavior. The Go-No-Go task that you are about to complete it a direct test of impulsivity. As you are a drinker, it is predicted that you will show more impulsive reaction times on this task.

Participants in the control condition were informed that the experiment was investigating the influence of contextual factors on inhibitory control. As such, they were not primed with any negative stereotype regarding the links between alcohol consumption and inhibitory control. Upon completion of the GNAT, participants completed two questions which allowed us to evaluate the effectiveness of the stereotype threat manipulation: 'To what extent are there differences in inhibition between drinkers and non-drinkers on this task?' $(1=$ No Difference and $10=$ Difference between drinkers and non-drinkers) and 'Who do you believe performs better on this task?' (1 = Non-drinkers, $10=$ Heavy drinkers $)$. Finally, participants completed the AUDIT and the ADQ to ensure that participants' drinking behavior and temperament did not differ as a function of experimental condition. These measures remained the final components to limit the signal strength of the study (Davies \& Best, 1996) and ensured that participants in the control condition did not experience stereotype threat.

\subsection{Data Analysis}

Statistical analyses were conducted using SPSS 22.0 (SPSS Inc., New York, USA). Exploratory analysis was first performed to ensure that data was normally distributed and that the assumption of homogeneity of variance had not been violated. A 2 (stimuli; alcohol, neutral) x 2 (sound cue; bar, none) mixed factorial Analysis of Variances (ANOVA) was conducted on accuracy data (proportion of correct responses), false accuracy alarm rate (incorrect responses) and response times. Only control participants' data was analysed to examine the influence of contextual cues on 
STEREOTYPE THREAT \& INHIBITORY CONTROL

inhibitory control (irrespective of stereotype threat). A 2 (condition; stereotype threat; non-stereotype threat) x 2 (stimuli; alcohol, neutral) x 2 (sound cue; bar, none) mixed ANOVA was then conducted to examine the effect of stereotype threat on participants' inhibitory control. Participants' self-reported drinking behavior and executive function did not differ as a function of experimental condition $(p>.05)$.

\section{Results}

\subsection{Stereotype Threat Manipulation Check}

An independent $t$-test revealed that participants in the stereotype threat condition more strongly endorsed that drinkers had limited inhibitory control $(M=7.40, S D=$ 1.39) relative to participants in the control condition $(M=4.55, S D=2.81), t(51)=$ 4.23, $p<.001, d=1.29$. Furthermore, participants in the stereotype threat condition $(M=2.35, S D=.92)$ were more likely to believe that non-drinkers performed better on the GNAT compared to control participants $(M=3.30, S D=1.42), t(51)=2.66, p$ $=.01, d=.79$.

\subsection{Standard instructions}

3.2.1. Response accuracy. A main effect of sound cue indicated that control participants responded to go-trials more accurately when bar-related sound was played $(M=.93, S D=.14)$ compared to no sound $(M=.91, S D=.14), F(1,34)=$ 19.19, $p<.001, \eta_{p}^{2}=.36$. There was no effect of experimental condition, $p>.05$.

3.2.2. False accuracy alarm rate (FAR). A main effect of stimuli on false alarm rate in no-go trials indicated that control participants made significantly less commission errors (false alarms) in response to alcohol-related cued targets $(M=.50$, $S D=.26)$ compared to neutral targets $(M=.65, S D=.18), F(1,34)=18.84, p<.001$, $\eta_{p}^{2}=.36$. A main effect of sound cue indicated that the presence of bar-related sound 
STEREOTYPE THREAT \& INHIBITORY CONTROL

increased commission errors $(M=.61, S D=.21)$ compared to when no sound was played $(M=.54, S D=.20), F(1,34)=16.08, p<.001, \eta_{p}^{2}=.32$. There was no effect of experimental condition, $p>.05$.

3.2.3. Response Latencies. A main effect of stimuli revealed that control participants were significantly faster to respond to the water bottles $(M=280.85, S D$ $=46.68)$ compared to alphabetical letters $(M=305.99, S D=39.40), F(1,34)=15.41$, $p<.001, \eta_{p}^{2}=.31$. A main effect of sound cue indicated that they were also significantly quicker to respond to go-stimuli when bar-related sound was presented $(M=285.64, S D=41.47)$ compared to no sound $(M=301.19, S D=37.39), F(1,34)$ $=43.02, p<.001, \eta_{p}^{2}=.56$. A main effect of experimental condition indicated that participants in the stereotype threat condition $(M=320.68, S D=177.03)$ were significantly slower to respond to go-targets compared to participants in the control condition $(M=293.42, S D=38.66), F(1,54)=6.54, p=.01, \eta_{p}^{2}=.12$

\subsection{Reverse Instructions}

3.3.1. Response accuracy. There was no main effect of stimuli or sound cue on control participants' accuracy rates, $p>.05$. However, an additive effect of experimental condition indicated that stereotype threatened participants were less accurate when responding to the beer bottle and letter ' $\mathrm{K}$ ' $(M=.81, S D=.22)$ relative to control participants $(M=.97, S D=.22), F(1,51)=14.54, p<.001, \eta_{p}^{2}=.22$. This was qualified by a three-way interaction between stimuli type, sound cue and experimental condition, $F(1,51)=25.61, p<.001, \eta_{p}^{2}=.33$. Stereotype threatened participants responded less accurately to alcohol-related stimuli in the presence of bar-related sound and no sound $(M=.85, S D=.29, M=.86, S D=.29$, respectively) compared to control participants $(M=.97, S D=.22, M=.97, S D=.22$ respectively), 


\section{STEREOTYPE THREAT \& INHIBITORY CONTROL}

$p<.05$. They also responded less accurately to neutral stimuli in the presence of barrelated sound and no sound $(M=.80, S D=.29, M=.75, S D=.22$, $)$ compared to control participants $(M=.97, S D=.22, M=.97, S D=.22$, respectively), $p=.001$. Furthermore, stereotype threatened participants responded more accurately to alcoholrelated stimuli compared to neutral stimuli in the presence of both bar-related sound $(M=.85, S D=.29, M=.80, S D=.29)$ and no sound $(M=.86, S D=.29, M=.75, S D$ $=.22$ respectively), $p<.05$.

3.3.2. FAR. There was no main effect of stimuli or sound cue on control participants' false responding rates, $p>.05$. An additive effect of experimental condition revealed that participants in the stereotype threat condition made significantly more errors $(M=.13, S D=.07)$ compared to the control condition $(M=$ $.01, S D=.07), F(1,45)=43.15, p<.001, \eta_{p}^{2}=.49$. This was qualified by a threeway interaction between experimental condition, stimuli type and sound cue, $F(1,45)$ $=27.32, p<.001, \eta_{p}^{2}=.38$. Stereotype threatened participants responded with more errors towards alcohol-related stimuli presented with bar-related sound $(M=.10, S D$ $=.14)$ and no sound $(M=.08, S D=.07)$ compared to participants in the control condition (both $M=.01, S D=.07$ ), $p<.001$. Stereotype threatened participants also made more errors when responding to neutral targets presented with bar-related sound $(M=.14, S D=.13)$ and no sound $(M=.19, S D=.12)$ compared to participants in the control condition $(\mathrm{M}=.01, \mathrm{SD}=.01), p<.001$.

3.3.3. Response Latencies. A main effect of stimuli revealed that control participants were significantly faster to respond to alcohol-related stimuli $(M=$ $372.87, S D=30.59)$ compared to neutral stimuli $(M=385.21, S D=29.79), F(1,32)$ $=12.05, p<.01, \eta_{p}^{2}=.27$. P. A main effect of sound cue also indicated that they were significantly faster to respond when a bar-related sound was played $(M=$ 
$374.18, S D=28.41)$ compared to no sound $(M=383.89, S D=29.39), F(1,32)=$ 27.75, $p<.001, \eta_{p}^{2}=.46$. A main effect of experimental condition indicated further that stereotype threatened participants took significantly longer to respond to the beer bottle and the letter ' $\mathrm{K}$ ' $(M=402.61, S D=48.05)$ compared to control participants $(M=379.04, S D=37.42), F(1,51)=7.95, p<.01, \eta_{p}^{2}=.14$.

\section{Discussion}

With the aim of examining the possible impact of stereotype threat on alcohol users' inhibitory control, participants were primed with the well-known stereotype that alcohol consumption has been linked to impulsive behavior. Here it was predicted that participants would exhibit diminished inhibitory control under stereotype threat. Results indicated that stereotype threatened participants were slower to respond to gotargets (water bottle, letter K) compared to control participants on standard instruction blocks. Furthermore, the addition of bar-related sound cues further diminished this dominant response, slowing stereotype threatened participants' responses to the water bottle, but not to the letter K. In reverse instruction trials, stereotype threatened participants responded slower and less accurately to both alcohol-related and neutral stimuli than control participants, and this was further enhanced by the addition of barrelated sound. However, in contrast to the control group, stereotype threatened participants responded more accurately to alcohol-related stimuli compared to neutral stimuli. Consistent with this finding, previous research indicates that individuals belonging to stigmatized groups may become vigilant to cues that highlight a discredited social identity (Kaiser et al., 2006). This increased vigilance may therefore enable people to detect, with the goal of avoiding, behavior that could be seen to confirm a negative stereotype (Jamieson \& Harkins, 2007). Resultantly, stereotype threatened participants may have responded to alcohol-related stimuli more accurately 
STEREOTYPE THREAT \& INHIBITORY CONTROL

in a bid to disconfirm the negative stereotype pertaining to drink-related impulsivity. Indeed, previous research has demonstrated the effects of stereotype threat on cannabis and ecstasy users' cognitive function (Cole et al., 2005; Looby \& Earleywine, 2010). As such, the current study extends these findings by transferring the 'stereotype threat' paradigm on illicit substances to a legal substance, namely alcohol.

A second aim of this research was to examine the contextual influences of alcohol-related stimuli and cues on inhibitory control. During standard instructions, participants were more accurate to respond to go-trials (water bottle, letter K) when bar-related sound was played compared to no sound. This suggests that the priming effects of bar-related sound facilitated response accuracy. Furthermore, false accuracy rates revealed that participants made fewer errors when responding to the water bottle compared to the letter $\mathrm{K}$, but the addition of bar-related sound increased errors compared to no sound. Taken together, these results suggest that bar-related sound facilitated responding in go-trials, but hindered performance on no-go trials. Moreover, participants also responded significantly faster to alcohol-related stimuli compared to neutral stimuli, with the addition of bar-related sound accelerating responding. In line with previous research (Fleming \& Bartholow, 2014; Rose \& Duka, 2008) quicker responses to alcohol-related targets may indicate these particular stimuli are more salient to social drinkers, attracting a greater amount of attention. Rather than indicating diminished inhibitory control, these results therefore suggest that individuals who drink alcohol may display an excitatory response approach towards alcohol-related cues. 


\subsection{Limitations and Recommendations for Future Research}

A number of limitations need to be borne in mind when interpreting the findings of the present study. Contrary to predictions, participants made more false errors when responding to neutral stimuli compared to alcohol-related stimuli. This may be explained by the ratio of go/no-go stimuli utilized for alcohol-related targets relative to neutral targets. Specifically, the ratio of go/no-go stimuli for alcoholrelated stimuli was 1:1, with participants either responding to a water bottle or beer bottle. However, the ratio for alphabetical letters was 25:1, with participants inhibiting a response to the letter $\mathrm{K}$ in comparison to other alphabetical letters. As such, inhibiting responses to neutral-targets may have been more difficult.

In the examination of stereotype threat, participants were explicitly primed that the study was investigating impulsivity and, accordingly, reaction times. It is therefore possible that this particular prime may have created an implicit demand for the participants to perform as expected (i.e., demand characteristics).

Furthermore, previous research has demonstrated that moderate doses of alcohol contribute to impaired inhibitory control tasks (Abroms \& Fillmore, 2004; Marczinski et al., 2005; Weafer \& Fillmore, 2014). In light of the current results, however, the proportion of variance attributable to stereotype threat - resultant from pre-test instructions of procedural signaling - as opposed to actual intoxication remains an interesting avenue for further elucidation. Future research should therefore investigate whether stereotype threat may be contributing to the observed deficits in inhibitory control mechanisms when individuals are intoxicated.

\subsection{Conclusion}

The acute effects of alcohol on inhibitory control mechanisms are widely studied, with research suggesting that alcohol alters the automatic processing of 
alcohol-related cues and impairs executive function. However, the current findings suggest that these cognitive responses, typically observed in laboratory studies, may be influenced by the knowledge of negative drink-related stereotypes. The current results indicate that although stereotype threatened participants launched slower responses to alcohol-related and neutral stimuli, they did so with reduced accuracy compared to the control group - a result consistent with impaired inhibitory control. Moreover, participants in the control condition displayed an attentional bias in the treatment of alcohol-related stimuli. These results may therefore have implications for public health campaigns and methodological designs with high procedural signaling with respect to not inadvertently inducing stereotype threat and thereby impacting alcohol-related behavior. Whilst it is important that individuals are aware of the negative consequences of alcohol consumption on cognitive functioning, it is conceivable that this information may be creating stereotypes about intellectual inferiority in the minds of alcohol drinkers. In order to reliably investigate the consequences of substance use, researchers should be cautious of how they outline experiments to participants, and disseminate their findings in the media. 
STEREOTYPE THREAT \& INHIBITORY CONTROL

\section{References}

Abroms, B. D., \& Fillmore, M. T. (2004). Alcohol-induced impairment of inhibitory mechanisms involved in visual search. Journal of Experimental and Clinical Psychopharmacology, 12, 243-250. doi: 10.1037/1064-1297.12.4.243

Adams, S., Ataya, A. F., Attwood, A. S., \& Munafò, M. R. (2013). Effects of alcohol on disinhibition towards alcohol related cues. Drug and Alcohol Dependence, 127, 137-142. doi: 10.1016/j.drugalcdep.2012.06.025

Clarke, N. C., Field, M., \& Rose, A. K. (2015). Evaluation of a brief personalized intervention for alcohol consumption in college students. PLoS One, 10, E0131229. Doi: 10.1371/journal.pone.0131229

Cole, J. C., Michailidou, K., Jerome, L., \& Sumnall, H. R. (2005). The effects of stereotype threat on cognitive function in ecstasy users. Journal of Psychopharmacology, 20, 518-525. doi: 10.1177/0269881105058572

Davies, J. B., \& Best, D. W. (1996). Demand characteristics and research into drug use. Psychology \& Health, 11, 291-299. doi: 10.1080/08870449608400258

Drink Aware. (2014) Health effects of alcohol: Alcohol and mental health. Available from: https://www.drinkaware.co.uk/check-the-facts/health-effects-ofalcohol/mental-health/alcohol-and-mental-health

Feldstein-Ewing, S. W., Sakhardande, A., \& Blakemore, S-J. (2014). The effect of alcohol consumption on the adolescent brain: A systematic review of MRI and fMRI studies of alcohol-using youth. Neuroimage: Clinical, 5, 420-437. doi: 10/1016/j.nicl.2014.06.011 Available at: https://www.drinkaware.co.uk/aboutus/research-and-impact/drinkaware-research/adolescent-brain-literature-review 


\section{STEREOTYPE THREAT \& INHIBITORY CONTROL}

Field, M., \& Cox, W. M. (2008). Attentional bias in addictive behaviors: A review of its development, causes, and consequences. Drug and Alcohol Dependence, 1, 120. doi: 10.1016/j.drugalcdep.2008.03.030

Field, M., \& Duka, T. (2002). Cues paired with a low dose of alcohol acquire conditioned incentive properties in social drinkers. Psychopharmacology, 159, 325-334. doi: 10.1007/s00213-001-0923-z

Fillmore, M. T. (2003). Drug abuse as a problem of impaired control: Current approaches and findings. Behavioral and Cognitive Neuroscience Reviews, 2, 179197. doi: $10.1177 / 1534582303257007$

Fleming, K. A., \& Bartholow, B. D. (2014). Alcohol cues, approach bias, and inhibitory control: Applying a dual process model of addiction to alcohol sensitivity. Psychology of Addictive Behaviors, 28, 85-96. doi: 10.1037/a0031565

Hogarth, L., Attwood, A. S., Bate, H. A., Munafò, M. R. (2012). Acute alcohol impairs human goal-directed action. Biological Psychology, 90, 154-160. doi: 10.1016/j.biopsycho.2012.02.016

Jamieson, J. P., \& Harkins, S. G. (2007). Mere effort and stereotype threat performance effects. Journal of Personality and Social Psychology, 93, 544-564. doi: $10.1037 / 0022-3514.93 .4 .544$

Jones, A., Rose, A. K., Cole, J., \& Field, M. (2013). Effects of alcohol cues on craving and ad libitum alcohol consumption in social drinkers: The role of disinhibition. Journal of Experimental Psychopathology, 4, 239-249. doi: $10.5127 /$ jep. 031912

Kaiser, C. R., Vick, S. B., \& Major, B. (2006). Prejudice expectations moderate preconscious attention to cues that are threatening to social identity. Psychological Science, 17, 332-338. 
STEREOTYPE THREAT \& INHIBITORY CONTROL

Kreusch, F., Vilenne, A., \& Quertemont, E. (2013). Response inhibition toward alcohol-related cues using an alcohol go/no-go task in problem and non-problem drinkers. Addictive Behaviors, 38, 2520-2528. doi: 10.1016/j.addbeh.2013.04.007

Looby, A., \& Earleywine, M. (2010). Gender moderates the impact of stereotype threat on cognitive function in cannabis users. Addictive Behaviors, 35, 834-839. doi: 10.1016/j.addbeh.2010.04.004

Marczinski, C. A., Abroms, B. D., Van Selst, M., \& Fillmore, M. T. (2005). Alcoholinduced impairment of behavioral control: Differential effects on engaging vs. disengaging responses. Psychopharmacology, 182, 452-459. doi: 10.1007/s00213005-0116-2

Monk, R. L., \& Heim, D. (2013). Environmental context effects on alcohol-related outcome expectancies, efficacy and norms: A field study. Psychology of Addictive Behaviors, 27, 814-818. doi: 10.1037/a0033948

Nosek, B. A., \& Banaji, M. R. (2001). The go/no-go association task. Social Cognition, 19, 625-666.

Rothbart, M. K., Ahadi, S. A., \& Evans, D. E. (2000). Temperament and personality: Origins and outcomes. Journal of Personality and Social Psychology, 78, 122-135. doi: $10.1037 / / 0022-3514.78 .1 .122$

Rose, A. K., \& Duka, T. (2008). Effects of alcohol on inhibitory processes. Behavioral Pharmacology, 19, 284-291. doi: 10.1097/FBP.0b013e328308f1b2

Saunders J. B, Aasland, O. G, Babor, T. F, De La Fuente, J. R, \& Grant, M. (1993). Development of the alcohol use disorders identification test (AUDIT): WHO collaborative project on early detection of persons with harmful alcohol consumption-II. Addiction, 88, 791-804. doi: 10.1111/j.13600443.1993.tb02093.x 
STEREOTYPE THREAT \& INHIBITORY CONTROL

Schmader, T., \& Johns, M. (2003). Converging evidence that stereotype threat reduces working memory capacity. Journal of Personality and Social Psychology, 85, 440-452. doi: 10.1037/0022-3514.85.3.440

Steele, C. M., \& Aronson, J. (1995). Stereotype threat and the intellectual test performance of African Americans. Journal of Personality and Social Psychology, 69, 797-811. doi: 10.1037/0022-3514.69.5.797

Wall, A. M., Mckee, S. A., \& Hinson, R. E. (2000). Assessing variation in alcohol outcome expectancies across environmental context: An examination of the situational-specificity hypothesis. Psychology of Addictive Behaviors, 14, 367-375. doi: 10.1037//0893-164x.15.3.219

Weafer, J., \& Fillmore, M. T. (2014). Alcohol-related cues potentiate alcohol impairment of behavioral control in drinkers. Psychology of Addictive Behaviors. doi: $10.1037 / \mathrm{adb} 0000013$ 